\title{
Vitamin A Deficiency and Pulmonary Oxygen Toxicity: Morphometric Studies in the Murine Lung
}

\author{
NICOLE COHEN-ADDAD, ROBERT BOLLINGER, JEAN CHOU, AND RONALD POLAND \\ Departments of Pediatrics [N.C.-A.] and Biostatistics [J.C.], UMDNJ/New Jersey Medical School, Newark, New \\ Jersey 07103; and Department of Pediatrics [R.B., R.P.], Wayne State University, Detroit, Michigan 48201
}

\begin{abstract}
Vitamin $A$ and its precursor $\beta$-carotene are naturally occurring antioxidants. The effects of diets deficient in $\beta$-carotene and vitamin $A$ on the alveolar-capillary membrane were studied in young adult BALB/C mice before and after exposure to $65 \%$ oxygen. One of three diets (standard complete, $\beta$-carotene deficient, or $\beta$-carotene and vitamin $A$ deficient) was fed for a 6 -wk period. Mice were then exposed to $65 \%$ oxygen for 0,3 , or 6 days, sacrificed, and their lungs examined by electron microscopy using the morphometric techniques of Weibel. The arithmetic and the harmonic mean thicknesses of the alveolarcapillary membrane and its components (epithelium, interstitium, and endothelium) were measured to assess the influences of diet and of duration of exposure to $65 \%$ oxygen. Analysis of variance and multiple comparisons of means (Student-Neuman-Keuls statistics) were applied. Diet alone did not significantly affect membrane thickness. However, duration of oxygen exposure increased the thicknesses of both the epithelium and interstitium in the group fed a diet deficient in both $\beta$-carotene and vitamin $A$ as compared to the other two groups although this was only significant for the epithelium. $\beta$-Carotene deficiency alone did not affect the respiratory membrane either before or after oxygen exposure. These results suggest that vitamin $A$ may be an important nutrient in the protection against pulmonary oxygen toxicity. (Pediatr Res 23: 76-80, 1988)
\end{abstract}

Host factors have long been thought to contribute to the incidence of pulmonary damage induced by noxious agents such as hyperoxia (1). The tissue concentrations of antioxidants may account for variability in host response. Some infants exposed to hyperoxia apparently escape its adverse effects while others develop bronchopulmonary dysplasia. Oxygen supplementation has been implicated as one of the main causes of bronchopulmonary dysplasia (2-6). Vitamin E deficiency worsens pulmonary damage from oxygen toxicity in animals $(7,8)$. Clinical trials with vitamin $E$ supplementation have failed to demonstrate protection against pulmonary oxygen toxicity in human newborn infants $(9,10)$. On the other hand, we have demonstrated in vitro that $\beta$-carotene is 100 times more effective than vitamin $E$ as an antioxidant $(11,12)$.

$\beta$-Carotene is a natural antioxidant without known toxicity even when administered in high doses (13). It is a precursor of vitamin $\mathrm{A}$ and converts to that vitamin as needed in humans

Received December 22, 1986; accepted September 16, 1987.

Correspondence Nicole Cohen-Addad, M.D., UMDNJ/New Jersey Medical School, 185 So. Orange Avenue, MSB-F-586, Newark, NJ 07103-2757. and other mammals such as mice. It quenches singlet oxygen and other free radicals which damage cell membranes (14). $\beta$ Carotene has also been cited recently as a possible protector against lung cancer in heavy smokers $(15-17)$, perhaps by quenching oxygen radicals (18). These effects, however, may be mediated through transformation of $\beta$-carotene into vitamin $\mathrm{A}$ which can independently inhibit lipid peroxidation (19). In vivo, $\beta$-carotene is a potent antioxidant therapeutic agent for erythropoietic protoporphyria (20).

The low serum $\beta$-carotene and vitamin A concentrations typical of human and especially premature neonates $(21,22)$ may predispose them to the toxic effects of oxygen. Preliminary results correlating low serum vitamin A concentrations and bronchopulmonary dysplasia have been reported recently $(23,24)$.

Unlike their newborn offspring seemingly resistant to pulmonary oxygen toxicity $(25,26)$, young adult mice exposed to supplemental oxygen manifest pulmonary oxygen changes that are similar to those of bronchopulmonary dysplasia. Exposure to $65 \%$ oxygen for $1 \mathrm{wk}$ has been shown to produce changes in animals made deficient in vitamin $E$ (5). Exposure of young adult mice ( $>4 \mathrm{wk}$ of age) to a diet deficient in $\beta$-carotene or a diet deficient in $\beta$-carotene and vitamin $\mathrm{A}$ for $6 \mathrm{wk}$ produces a significant decrease in lung retinol concentrations. Mice exposed to any of the aforementioned diets for such a period do not exhibit any sign of vitamin A deficiency.

We therefore conducted a prospective control study of the effects of diets deficient in $\beta$-carotene and vitamin $A$ on the lung morphology of mice subsequently exposed to oxygen supplementation over time. The objectives of this study were also to examine the importance of $\beta$-carotene in the maintenance of the respiratory membrane and to distinguish its role from that of its metabolite, vitamin $\mathrm{A}$.

\section{MATERIALS AND METHODS}

Young adult female BALB/C mice were randomly divided into three groups assigned for 6 wk to: 1) standard complete, 2) $\beta$-carotene deficient, or 3) $\beta$-carotene- and vitamin A-deficient diets. All diets were obtained from ICN Nutritional Biochemicals, Cleveland, $\mathrm{OH}$. The standard complete diet consisted of $27 \%$ vitamin-free casein, $59 \%$ starch, $10 \%$ cottonseed oil, $4 \%$ salt mixture (USP-IV), and ICN vitamins. The $\beta$-carotene-deficient diet was made by replacing the cottonseed oil with lard, which is poor in $\beta$-carotene. ICN vitamins containing vitamin $\mathrm{A}$ but no $\beta$-carotene were added to this second diet. The diet deficient in $\beta$-carotene and vitamin A was identical to the second diet except that vitamin $A$ and $\beta$-carotene were absent from the vitamin mixture. The $\beta$-carotene content in the standard diet was $3 \mu \mathrm{g} / \mathrm{kg}$ diet. The vitamin A content in diets 1 and 2 was $39.6 \mathrm{mg}$ retinyl acetate $/ \mathrm{kg}$ diet $(500,000 \mathrm{IU} / \mathrm{g})$. There was no detectable $\beta$-carotene in diets 2 or 3 and no detectable vitamin 
$A$ in diet 3 (measurements done by high-performance liquid chromatography).

The mice were housed in cages placed under bell-type oxygen chambers and fed ad libitum throughout the experiment. Each oxygen chamber contained two cages with a maximum of five mice per cage. The oxygen concentration in the chambers was maintained at $65 \pm 2 \%$ and monitored every $12 \mathrm{~h}$. The humidity inside the chambers was $80 \pm 2 \%$. The $\mathrm{CO}_{2}$ content inside the chambers was monitored daily and was kept at $0.20 \pm 0.09 \%$ through the use of $\mathrm{CO}_{2}$ absorbents.

At the end of the 6-wk period on one of the three diets, nine mice from each group were equally subdivided and randomly selected for sacrifice after 0,3 , and 6 days of exposure to $65 \%$ oxygen. Lung sections were harvested for electron microscopy in the following manner: 1) intraperitoneal injections of phenobarbital $(50 \mathrm{mg} / \mathrm{kg}), 2)$ tracheostomy with insertion of a polyethylene cannula (Intramedic no. 1190), 3) transabdominal incisions to create pneumothoraces, 4 ) infusion of $0.1 \mathrm{M}$ cacodylate buffered $2 \%$ glutaraldehyde into the tracheobronchial tree under $20 \mathrm{~cm}$ of water pressure for $5 \mathrm{~min}$, and 5) removal of the lungs intact. The lung tissues were then processed through a fixative wash and holding buffers using standard techniques and embedded in Epon.

The electron microscopy measurements of the alveolar-capillary membrane were performed by hand and according to Weibel's techniques (27-29). Sections were picked up on 400-mesh copper grids and stained with uranyl acetate and lead citrate. A technically acceptable section, near to the center of the copper grid, was selected for each block. The sections typically occupied 30 to 40 grid squares. A multipurpose lattice inscribed on the electron microscope screen was used for measurements of tissue point density and barrier thickness (27). This lattice was superimposed on the electron image at each corner of the copper grid square. At the magnification used, $\times 7300$, this yielded four nonoverlapping fields per copper grid square. Prior to each session, the microscopy was calibrated with a cross-grating replica (2160 lines/mm).
Endothelial, interstitial, and epithelial percentages were estimated by point density counts using the end points of the lattice lines. Arithmetic barrier thickness was estimated by tissue point density counts and intersections of the inner and outer surfaces of the anatomical barrier with the lattice lines. The harmonic mean barrier thickness was estimated by linear measurements of the barrier intercepts using linear rows of dots as suggested by Weibel. Two tissue blocks per animal were selected randomly and a total of 150 to 250 fields were studied per block.

The arithmetic and harmonic mean thickness of the respiratory membrane and its components (epithelium, interstitium, and endothelium) were measured using Weibel's grid statistics. The arithmetic mean thickness emphasizes the contribution of the thicker portions of the respiratory membrane especially the junctional areas between alveoli. The harmonic mean thickness emphasizes changes in the thinner, functional portions of the respiratory membrane, which reflect those areas most responsible for gas exchange. Comparisons of the mean measurements were made using analysis of variance followed by the multiple comparison of means (Student-Neuman-Keuls procedure) when appropriate. In addition, descriptive histology was performed by electron microscopy and by light microscopy on other lung specimens from identically treated animals.

\section{RESULTS}

The results of the morphometric measurements are summarized in Figures 1 and 2. Prior to exposure to 65\% oxygen, the mean thicknesses of the respiratory membrane and its components showed no significant differences between groups after 6 wk on one of the three dietary regimens.

The means of the morphometric measurements were then compared over time of exposure to $65 \%$ oxygen to evaluate the additive effects of oxygen supplementation to the dietary differences. No significant change in mean thicknesses was detected in any of the dietary groups before day 6 of oxygen exposure. At day 6 , the arithmetic mean thickness of all three components of

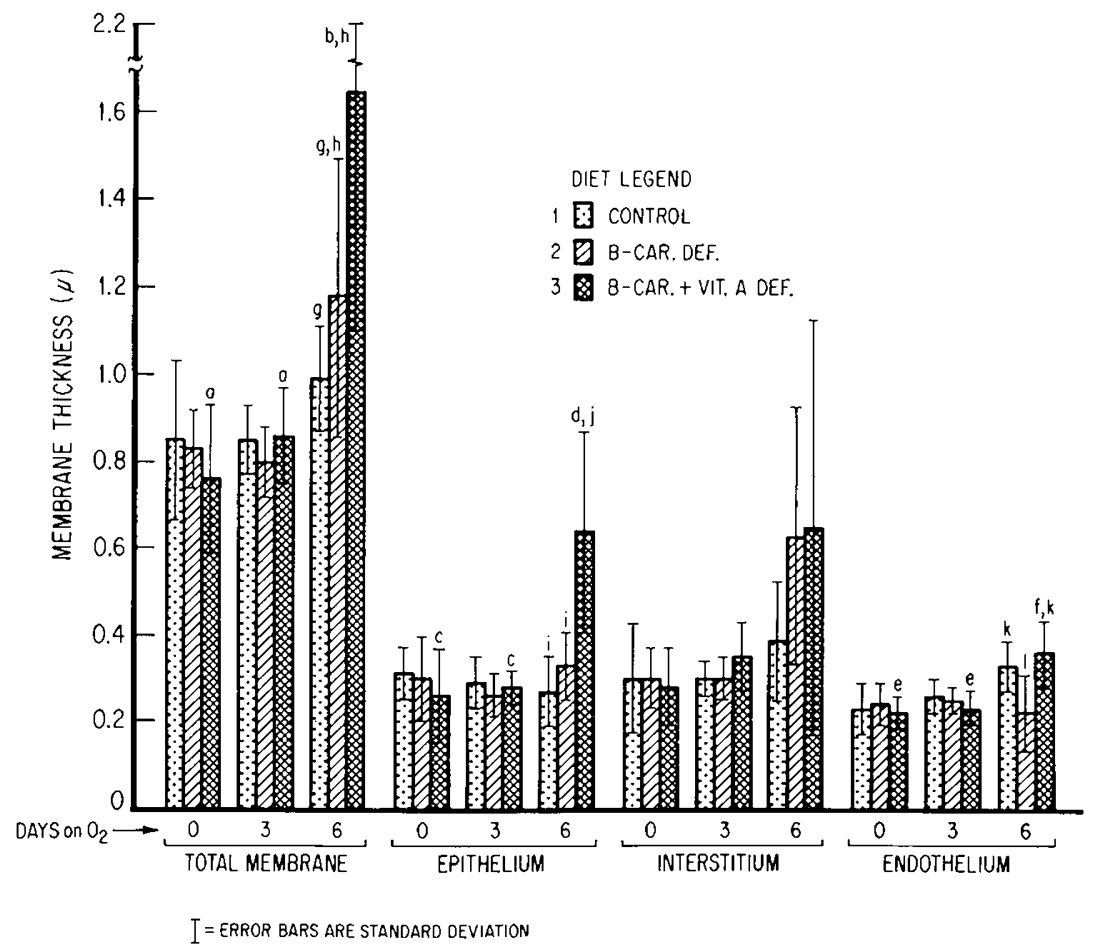

Fig. 1. Arithmetic mean thickness of the various components of the respiratory membrane. Statistical difference $(\alpha$ level of 0.01$)$ was observed only among values connoted with letters. In this group, bars with the same letter are not significantly different from each other, those with different letters are significantly different. 


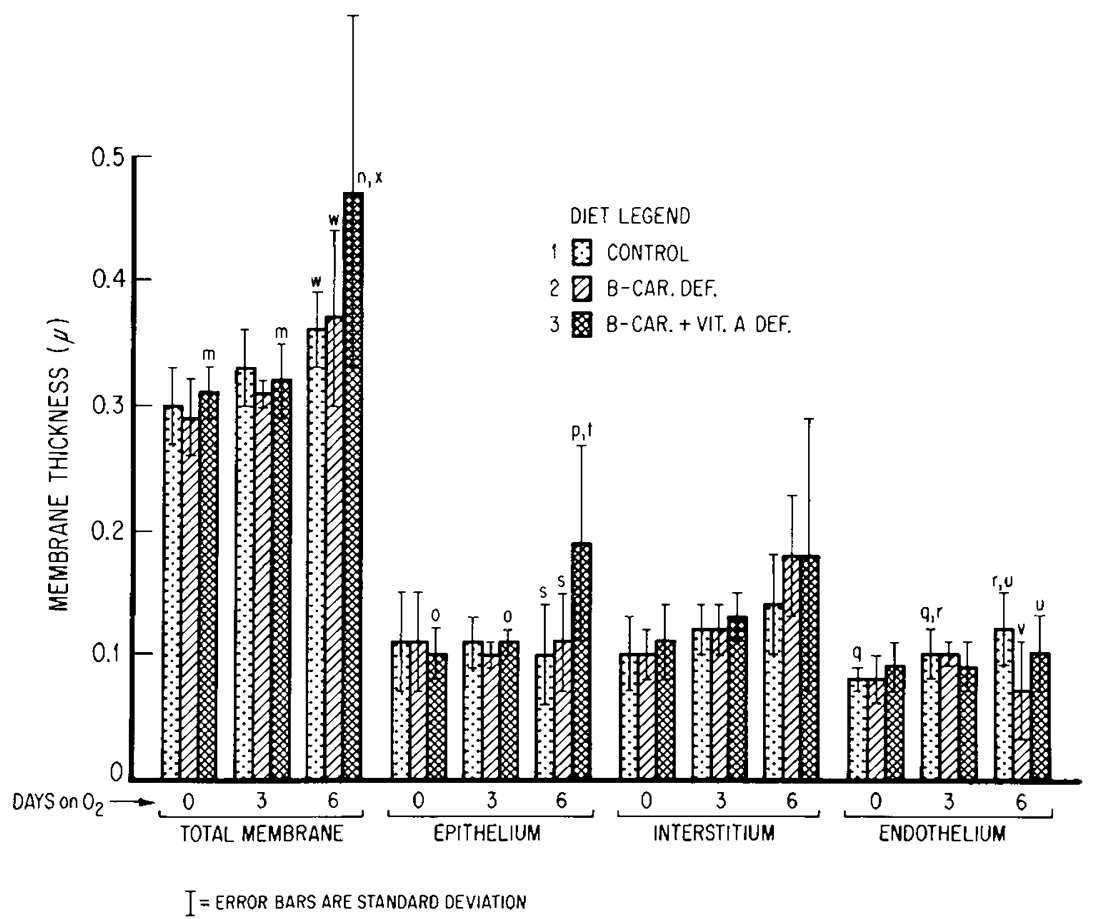

Fig. 2. Harmonic mean thickness of the various components of the respiratory membrane. Statistical difference ( $\alpha$ level of 0.01$)$ was observed only among values connoted with letters. In this group, bars with the same letter are not significantly different from each other, those with different letters are significantly different.

the respiratory membrane was increased in those animals made deficient in both $\beta$-carotene and vitamin A when compared to the other two diet groups. However, these increases in mean thickness were large enough to be statistically significant only in the epithelial component $(p<0.01)$. This difference was related primarily to a swelling and degeneration of type I respiratory epithelial cells. The increase in the arithmetic mean thicknesses of the respiratory membrane components translated into a statistically significant increase in the mean thickness of the total respiratory membrane on day 6 for the groups subjected to the diet deficient in both $\beta$-carotene and vitamin $A$.

For the harmonic mean thickness, the epithelial component and the total membrane both showed a statistically significant increase at day 6 for the $\beta$-carotene- and vitamin A-deficient group compared to the other two groups. The interstitial component showed a slight, although not statistically significant increase, and the endothelial component remained essentially the same.

Within the $\beta$-carotene- and vitamin A-deficient diet group, if the duration of exposure to oxygen is examined, the most obvious increase in both the arithmetic and harmonic mean thickness is again in the epithelial component. There was a statistically significant increase in the epithelial mean thickness between day 6 on one side and days 3 and 0 on the other side in the animals subjected to that diet. This relationship was also true for the arithmetic mean thickness of the endothelium and the harmonic and arithmetic mean thicknesses of the total membrane.

While not statistically significant, there was, however, an increase in mean thickness of the interstitium on day 6 of oxygen exposure for the $\beta$-carotene-deficient vitamin A-sufficient diet group compared to days 0 and 3 for the arithmetic and harmonic mean thicknesses. This increase translated into an increase in mean thickness of the total respiratory membrane for the arithmetic and harmonic mean thickness, although again this increase did not reach statistical significance.

There was seemingly no increase in mean thickness for any of the components of the respiratory membrane with exposure to oxygen, whether at day 3 or day 6 , for the $\beta$-carotene- and vitamin A-sufficient group. Only the endothelial harmonic mean thickness showed a mild increase at day 6 over day 0 .

A comparison of values between diet groups at each interval of oxygen exposure showed no difference in mean thickness for any of the components of the respiratory membrane at day 0 or 3 . On day 6 of oxygen exposure an increase in mean thickness was observed for the epithelial and interstitial components and translated into an increase in the total respiratory membrane mean thickness for the $\beta$-carotene- and vitamin A-deficient diet compared to the other two diet groups, both for arithmetic and harmonic mean thickness. These increases were, however, statistically significant only for the epithelial component, both arithmetic and harmonic, and for the total respiratory membrane mean thickness-only the harmonic mean thickness.

The descriptive histology by electron microscopy supported the aforementioned quantitative findings. For day 0 , the sections from lungs on either of the three diets appeared the same. The endothelial and epithelial cells were thin and dense, there was no platelet or erythrocyte adherence, and the fibrosis was limited to intercapillary bridges. In addition, there was minimal pinocytosis, the intercellular junctions appeared normal, and there were few, small, well-formed mitochondria.

On day 3 , the deficient diets began to produce changes in the histology of the lung. In those animals on the $\beta$-carotene- and vitamin A-sufficient diet, the lung structure remained the same with the exception that there was some platelet adherence. The lung tissue of those animals on the $\beta$-carotene-deficient vitamin A-sufficient diet showed slight changes. Most of the epithelial and endothelial cells were thin and dense, but some were pale and swollen. A few intercellular junctions were open. There was still minimal pinocytosis and small well-formed mitochondria. Lung sections from the animals on the $\beta$-carotene- and vitamin A-deficient diet showed the epithelium, endothelium, and interstitium to be much thicker and hypercellular. The cytoplasm was dense, and there was some fibrosis and edema. Junctions, mitochondria, and minimal pinocytosis remained the same.

By day 6 , even the lung tissue of those animals on the sufficient diet was beginning to show pale and swollen endothelial and 
epithelial cells. There was also evidence of minimal interstitial fibrosis and edema, some epithelial necrosis, and moderate pinocytosis. However, the mitochondria were still small and well formed and the junctions were normal. The $\beta$-carotene-deficient vitamin A-sufficient diet group showed thickening and edema of the endothelium, some epithelial necrosis, and an increase in thickness of the interstitium due to edema. The mitochondria were large, many junctions were open, and there was active pinocytosis. The lung tissue of the animals on the $\beta$-caroteneand vitamin A-deficient diet showed the endothelium and epithelium to be swollen and pale. In addition, the epithelium was often necrotic. The interstitium was hypercellular with a tortuous, duplicated, broad basement membrane, and exhibited more severe edema. There was platelet and erythrocyte adherence, and the junctions were open.

With the light microscope, only the gross effects of diet and oxygen exposure were visible. However, histological differences between the $\beta$-carotene- and vitamin A-deficient diet and the normal diet at day 6 could be distinguished (Fig. 3 ). The sections of the lungs from animals on the normal diet had very few red blood cells outside of the blood vessels. There was no evidence of edema and the membranes were intact. By contrast, lung sections from the $\beta$-carotene- and vitamin A-deficient animals showed many red blood cells in the alveolar spaces. There was also evidence of edema in the interstitium and many membranes were no longer intact.

\section{DISCUSSION}

We have shown that $6 \mathrm{wk}$ of exposure to diets deficient in either $\beta$-carotene and vitamin $\mathrm{A}$ or $\beta$-carotene alone are not associated with significant differences in thickness of the components of the respiratory membrane using Weibel's morphometric techniques. In addition, we have demonstrated that a dietary deficiency of both vitamin $\mathrm{A}$ and its precursor, $\beta$-carotene, is associated with a thickening of the epithelial portion of the respiratory membrane after exposure to $65 \%$ oxygen over a 6-day period as compared to a control diet and to a diet deficient only in $\beta$-carotene. We interpret this increase in thickness as an early result of oxygen toxicity to a membrane with impaired defenses to the toxic effects of oxygen. When $\beta$-carotene was withheld but vitamin A supplied in the diet, respiratory epithelial thickness was comparable to that of the control (complete) diet. Therefore, the differences in host defense were inferentially related to the lack of vitamin A rather than that of its precursor, $\beta$-carotene.

Whereas the statistically significant differences were observed essentially for the epithelial component of the respiratory membrane, there was a progressive increase in mean thickness of the interstitium for both deficient diet groups over time of oxygen exposure. Electron micrographs confirmed the results by revealing the increasingly edematous aspect of the interstitium in the two diet groups on days 3 and 6 and, in addition, in the control group on day 6 . These interstitial findings further substantiate the toxic effects of oxygen supplementation.

While some other species, such as the hamster, show susceptibility to oxygen toxicity from the newborn period, mice were chosen for this study because a large source of data have already been accumulated regarding oxygen toxicity within this species. In addition, an "adult" model avoids the interference of variables related to immaturity and variability due to maturational changes, making it a "cleaner" model for the study of nutritional variables in relation to oxygen exposure. Finally, bronchopulmonary dysplasia is seen mostly in infants past the neonatal period and our mice were $6 \mathrm{wk}$ postweaning at the time of the study. The swelling and degeneration observed on the type 1 respiratory epithelial cells of the $\beta$-carotene- and vitamin Adeficient diet at day 6 of oxygen exposure could not have been related to poor fixation since all lungs were fixed immediately,

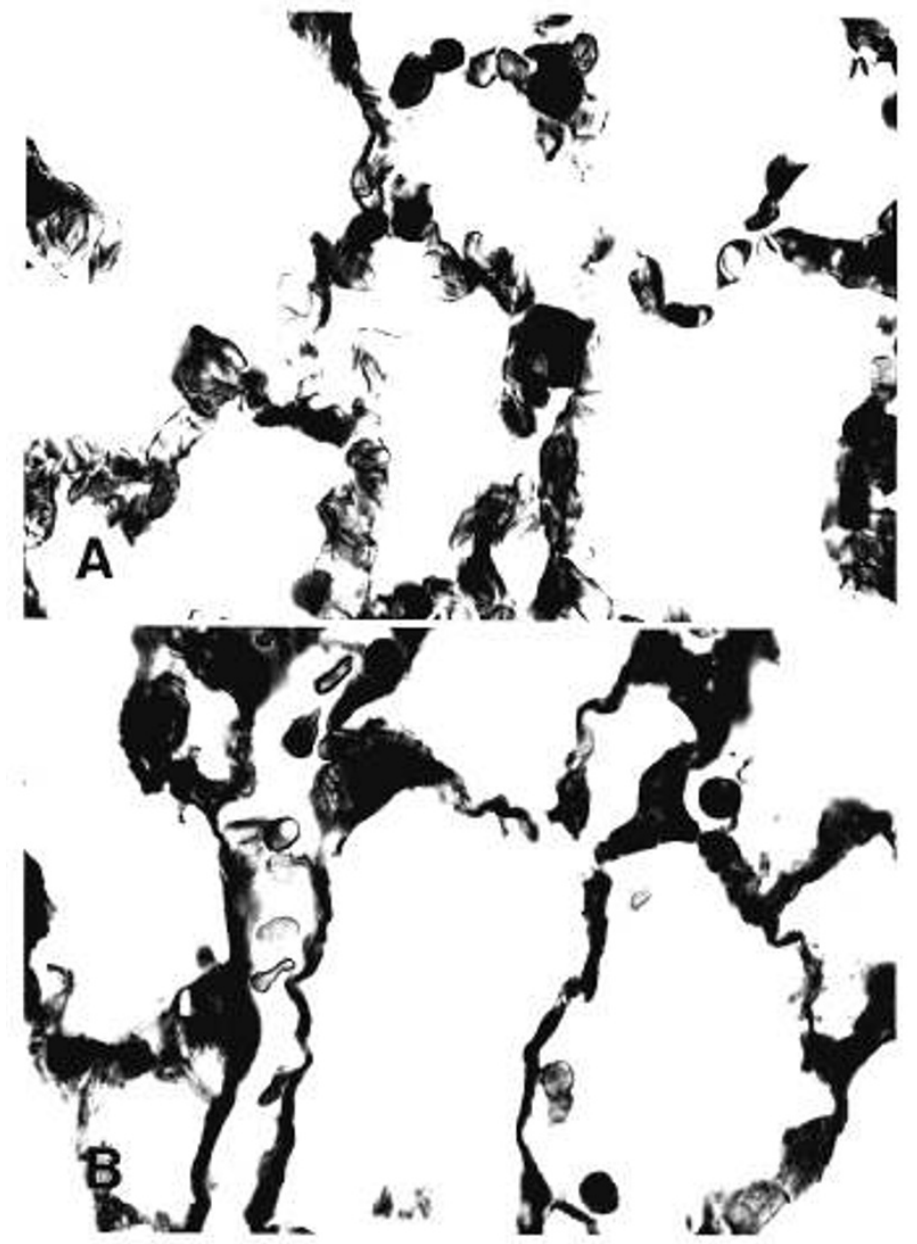

Fig. 3. Light microscope photomicrographs of lung tissue from mice exposed to $65 \%$ oxygen for 6 days. $A$, animal on the control diet. $B$, animal on the $\beta$-carotene- and vitamin A-deficient diet.

processed according to the same time schedule with the same fixatives and buffers, and similar histological changes were not observed in the epithelial cells of the other groups.

The source of fat used in the three diets, with subtle differences in fatty acid composition or in other associated contents (e.g. minerals), did not influence the results since the fat composition of the two "deficient" diets was identical and only the diet made deficient in both vitamin $A$ and $\beta$-carotene showed the effects of oxygen. If the differences in fat source had been influential one would have expected to observe differences in results between the control group and the $\beta$-carotene-deficient group. Tocopherol lung and liver concentrations measured in another study of similar design (Cohen-Addad $\mathrm{N}$, Tangney $\mathrm{C}$, unpublished data) were found similar between groups. Thus, vitamin $E$ status was unlikely to be a variable in this study. We are unable to test the effects of vitamin A deficiency independent of $\beta$-carotene deficiency because a diet containing $\beta$-carotene and no vitamin $A$ will allow the animal to synthesize vitamin $\mathrm{A}$ from the $\beta$-carotene present in the diet in order to prevent deficiency of the vitamin (30). This question could be addressed if we could find a technique that would block the conversion of $\beta$-carotene to vitamin A.

A difference in the morphometry of the respiratory membrane related to vitamin $A$ and $\beta$-carotene deficiency occurred only at the conclusion of 6 days of $65 \%$ oxygen exposure, and probably represents only the earliest stages of oxygen toxicity related to vitamin A deficiency. It would be helpful in future studies to prolong the time course to see whether other portions of the respiratory membrane show changes related to diet and oxygen 
and to see whether the differences are absolute or due to the timing of oxygen toxicity.

We conclude that vitamin A deficiency is associated with an enhanced susceptibility to oxygen damage manifested by thickening of the epithelial layer of the respiratory membrane, primarily the type I alveolar cell. Absence of $\beta$-carotene in the diet alone does not lead to similar oxygen damage when vitamin $A$ is supplied to the animals. These observations may provide some insight into the host variability in susceptibility to oxygen toxicity that we have observed in the newborn intensive care unit. There may eventually be a role for vitamin A or $\beta$-carotene supplementation for high risk newborn infants. However, more experimental evidence for this hypothesis will be needed before any such recommendation can be made.

Acknowledgments. The authors acknowledge the technical assistance of Pat Mathiew for the electron microscopy preparations and of Dr. Hugh Evans for his review of the manuscript.

\section{REFERENCES}

1. Clark JM, Lambertsen CJ 1971 Pulmonary oxygen toxicity: a review. Pharmacol Rev 23:37-132

2. Berg TJ, Pajtokhan RD, Reed MH, Langston C, Chernick V 1975 Bronchopulmonary dysplasia and lung rupture in hyaline membrane disease: influence of continuous distending pressure. Pediatrics 55:51-54

3. Deneke SM, Fanburg BL 1980 Normobaric oxygen toxicity of the lung. N Engl J Med 303:76-86

4. Crapo JD, Peters-Golden M, Marsh-Salin J, Shelburne JS 1978 Pathologic changes in the lungs of oxygen-adapted rats. Lab Invest 39:640-653

5. Nash G, Blennerhassett JB, Pontoppidian H 1967 Pulmonary lesions associated with oxygen therapy and artificial ventilation. N Engl J Med 276:368-374

6. Ludwin SK, Northway WH, Bensch KG 1974 Oxygen toxicity in the newborn. Lab Invest 31:425-435

7. Mino M 1973 Oxygen poisoning and vitamin E deficiency. J Nutr Sci Vitaminol 19:95-104

8. Poland RL, Bollinger RO, Bozynski ME, Karna P, Perrin EVD 1977 Effect of vitamin E deficiency on pulmonary oxygen toxicity. Pediatr Res 11:577

9. Ehrenkranz RA, Ablow RC, Warshaw JB 1979 Prevention of bronchopulmonary dysplasia with vitamin $\mathrm{E}$ administration during the acute stages of respiratory distress syndrome. J Pediatr 85:873-878

10. Saldanha RL, Cepeda EE, Poland RL 1982 The effect of vitamin E prophylaxis on the incidence and severity of bronchopulmonary dysplasia. J Pediatr 101:89-93

11. Cohen-Addad NE, Ostrea EM Jr 1979 Inhibitors of photodynamic oxidation: a comparative study. Am Soc Photobiol 7:89

12. Cohen-Addad NE, Ostrea EM Jr, Poland RL $1980 \beta$-carotene, vitamin A and photo-oxidation of bilirubin: mechanisms of action. Am Soc Photobiol 8:103

13. Bagdon RE, Zbinden G, Studer A 1960 Chronic toxicity studies of $\beta$-carotene. Toxicol Appl Pharmacol 2:225-236

14. Krinsky NI 1979 Carotenoid protection against oxidation. Pure Appl Chem 51:649-659

15. Cohen MH, Primack A, Broder LE, Williams LR 1977 Vitamin A serum levels and dietary vitamin $A$ intake in lung cancer patients. Cancer Lett 4:51-54

16. 1980 Vitamin A, retinol, carotene and cancer prevention. Br Med J 281:957958

17. Shekelle RB, Liu S, Raynor WJ Jr, Lepper M, Maliza C, Rossof AH 1981 Dietary vitamin A and risk of cancer in the Western Electric study. Lancet 2:1185-1198

18. Ames BN 1983 Dietary carcinogens and anticarcinogens: oxygen radicals and degenerative diseases. Science 221:1256-1264

19. Kartha VNR, Krishanamurthy S 1977 Antioxidant function of vitamin A. Int J Vit Nutr Res 47:394-401

20. Mathews-Roth MM, Pathak MA, Fitzpatrick TB, Harber LC, Kass EH 1970 Beta-carotene as a photoprotective agent in erythropoietic protoporphyria N Engl J Med 282:1231-1234

21. Baker H, Frank O, Thomson AD, Langer A, Munves ED, DeAngelis B, Kaminetzky HA 1975 Vitamin profile of 174 mothers and newborns at parturition. Am J Clin Nutr 28:59-65

22. Shenai JP, Chytil F, Jhaveri A, Stahlman MT 1981 Plasma vitamin A and retinol-binding protein in premature and term neonates. J Pediatr 99:302305

23. Hustead VA, Gutcher GR, Anderson SA, Zachman RD 1984 Relationship of vitamin A (retinol) status to lung disease in the preterm infant. J Pediatr 105:610-615

24. Shenai JP, Chytil F, Stahlman M 1985 Vitamin A status of neonates with bronchopulmonary dysplasia. Pediatr Res 19:185-188

25. Frank L, Bucher JR, Roberts RJ 1978 Oxygen toxicity in neonatal and adult animals of various species. J Appl Physiol 45:699-704

26. Polgar G, Antagnoli W, Ferrigan LW, Martin EA, Gregg WP 1966 The effect of chronic exposure to $100 \%$ oxygen in newborn mice. Am $J$ Med Sci 252:580-587

27. Weibel ER, Knight BW 1974 Morphometric study on the thickness of the pulmonary air-blood barrier. J Cell Biol 21:367-384

28. Gehr P, Bachofen M, Weibel ER 1978 The normal human lung: Ultrastructure and morphometric estimation of diffusion capacity. Respir Physiol 32:121140

29. Weibel ER 1979 Morphometry of the human lung: the state of the art after two decades. Bull Eur Physiopathol Respir 15:999-1013

30. Brubacher GB, Weiser H 1985 The vitamin A activity of $\beta$-carotene. Internat $\mathrm{J}$ Vit Nutr Res 55:5-14 